\title{
CPMG experiments for Protein Minor Conformer Structure Determination
}

Anusha B Gopalan ${ }^{1,{ }^{\star}}$, D Flemming Hansen ${ }^{2,{ }^{\star}}$, Pramodh Vallurupalli ${ }^{1,{ }^{\star}}$

${ }^{1}$ TIFR Centre for Interdisciplinary Sciences, 21 Brundavan Colony, Narsingi, Hyderabad, 500075, (India) E-mail: anushabg@tifrh.res.in, pramodh@tifrh.res.in

${ }^{2}$ Division of Biosciences, Institute of Structural and Molecular Biology, University College London, Gower Street, London, WC1E 6BT (UK) E-mail: d.hansen@ucl.ac.uk

${ }^{\star}$ Corresponding authors

Running Title: CPMG experiments to study protein dynamics. 
CPMG experiments to study protein dynamics

\begin{abstract}
CPMG relaxation dispersion NMR experiments have emerged as a powerful method to characterize protein minor states that are in exchange with a visible dominant conformation, have lifetimes between $\sim 0.5$ and 5 milliseconds (ms) and populations greater than $0.5 \%$. The structure of the minor state can, in favorable cases, be determined from the parameters provided by the CPMG relaxation dispersion experiments. Here we go through the intricacies of setting up these powerful CPMG experiments.
\end{abstract}

Keywords NMR, Protein, Conformational Dynamics, CPMG, Relaxation Dispersion 


\section{Introduction}

Under ambient conditions in aqueous solutions protein molecules interconvert between different conformational states that can have different populations and lifetimes [1-3]. Exchange is quite often between a dominant major state and other transiently populated minor states $[4,5]$. The populations of the states depend on their relative free energies while their lifetimes is largely dependent on the size of the free energy barrier that separates them from other states [6]. Peaks in NMR spectra arising from these minor conformers are usually not visible due to their low populations and short lifetimes and these minor conformers have to be detected by manipulating the visible major state magnetization [7]. Minor conformers that have lifetimes between 0.5 and 5 milliseconds (ms) can be detected using the CarrPurcell-Meiboom-Gill (CPMG) type relaxation dispersion experiments. In the constant time (CT) version of the CPMG experiment, transverse magnetization is allowed to evolve for a fixed time $T_{C P M G}$, during which refocusing $\pi\left(180^{\circ}\right)$ pulses are applied [8]. The transverse magnetization can be of the single quantum or multi quantum variety and can originate from different nuclei like ${ }^{1} \mathrm{H},{ }^{13} \mathrm{C}$ and ${ }^{15} \mathrm{~N}[9,10]$. When there is chemical exchange in the millisecond timescale regime the intensity of the visible state peak depends on the frequency $v_{C P M G}$ at which the $\pi$ pulses are applied. Here $v_{C P M G}=\frac{1}{4 \tau_{C P M G}}$ where $2 \tau_{C P M G}$ is the time between two $\pi$ pulses. The effective relaxation rate $R_{2, \text { eff }}\left(v_{C P M G}\right)=-\frac{1}{T_{C P M G}} \ln \left(\frac{I\left(v_{C P M G}\right)}{I_{0}}\right)$ depends on $v_{C P M G}$ thereby giving rise to the relaxation dispersion curve. Here $I\left(v_{C P M G}\right)$ is the peak intensity at a given $v_{C P M G}$ value and $I_{0}$ is the peak intensity in the reference experiment recorded in the absence of the $T_{C P M G}$ delay. The minor state population, lifetime and chemical shift can be obtained by analyzing the relaxation dispersion curve(s) [7,11].

In this chapter we describe how to setup NMR CPMG experiments that probe the dynamics at different protein backbone sites. We also provide a very limited overview on how to analyze the data.

\section{$2 \quad$ Materials}

2.1 NMR Samples: Protein samples with different isotope labeling schemes are required to study the dynamics at various protein backbone positions [12]. These include i) A uniformly ${ }^{15} \mathrm{~N} /{ }^{13} \mathrm{C} /{ }^{2} \mathrm{H}$ enriched sample. ii) A uniformly ${ }^{15} \mathrm{~N} /{ }^{13} \mathrm{C} / 50 \%{ }^{2} \mathrm{H}$ enriched sample. iii) $\mathrm{A}{ }^{15} \mathrm{~N} /{ }^{13} \mathrm{C} \alpha$ labeled sample. Sample (i) is used to study dynamics at the amide $\left({ }^{1} \mathrm{H},{ }^{15} \mathrm{~N}\right)$ and carbonyl $\left({ }^{13} \mathrm{C}\right)$ positions. Sample (ii) is used to study dynamics at $\mathrm{H} \alpha$ position. Sample (iii) is used to 
study dynamics at the $\mathrm{C} \alpha$ position. Although not necessary it is preferable to use a uniformly ${ }^{15} \mathrm{~N} /{ }^{2} \mathrm{H}$ enriched sample for amide ${ }^{15} \mathrm{~N} /{ }^{1} \mathrm{H}$ single quantum (SQ), zero quantum (ZQ) and double quantum (DQ) CPMG experiments. Preliminary experiments to standardize buffer conditions, temperature etc. can be performed using just a ${ }^{15} \mathrm{~N}$ enriched sample. The concentration of protein samples used in CPMG studies are usually between 1 to 2 millimolar $(\mathrm{mM})$. Experiments can also be performed with $0.5 \mathrm{mM}$ samples when the spectrometer is equipped with a cryogenically cooled probe.

2.1 NMR Spectrometers: The experiments are usually performed on spectrometers equipped with triple resonance probes. The minimum magnetic field strength used is $11.7 \mathrm{~T}$ (500 $\mathrm{MHz}$ ${ }^{1} \mathrm{H}$ resonance frequency). Experiments are usually performed at two well-separated field strengths like $11.7 \mathrm{~T}$ and $16.5 \mathrm{~T} / 18.8 \mathrm{~T}$ (500 and 700/800 MHz).

2.2 Thermocouple for temperature measurement: A thermocouple with a long $(\sim 3 \mathrm{~m})$ wire connecting it to a digital display is inserted into a standard NMR sample tube. The thermocouple is positioned where the sample center would be and the wire coming out of the top of the tube is attached to the tube using Parafilm to hold the thermocouple in place. The tube can now be inserted into a spinner assembly and loaded into the magnet in the usual manner to measure the sample temperature.

\section{$3 \quad$ Methods}

3.1 Sample Temperature Measurement and Setting: Temperature affects the kinetics of the processes being studied [6] and has to be kept constant during all the experiments. To keep the sample temperature constant between different spectrometers it is important to measure the sample temperature in each of them for a given variable temperature (VT) setting.

1. Measure the sample temperature for a given VT setting. The sample temperature can be easily measured using a thermocouple inserted into a standard sample tube as described in section 2.2 or using temperature standards like methanol (See Note 1).

2. Adjust the setting of the VT unit till the desired temperature is obtained. 
3.2 Setting the ${ }^{1} \mathbf{H}$ carrier positions: In most of the experiments described here the ${ }^{1} \mathrm{H}$ radiofrequency $(\mathrm{RF})$ carrier is centered on the water signal. In water samples the water resonance can be quite broad $(\sim 100 \mathrm{~Hz})$ on a typical triple resonance probe and the peak center cannot be determined accurately. A simple presaturation procedure can be used to obtain the water resonance frequency.

1. Obtain an estimate of the water resonance frequency from a one scan 1D spectrum obtained using a small flip angle $\left(\sim 10-15^{\circ}\right)$ pulse applied at the highest allowed power (usually $25-50 \mathrm{kHz}$ ).

2. Array the ${ }^{1} \mathrm{H}$ carrier frequency in $1 \mathrm{~Hz}$ steps around the estimated offset from step 1 and record a series of $1 \mathrm{D}$ experiments with a weak 2 second presaturation applied at a very low $B_{1}$ field of $\sim 100 \mathrm{~Hz}$ followed by the small flip angle pulse applied at full power. Process each recorded FID in an absolute value (magnitude) mode. The spectrum with the lowest intensity water peak is obtained when the carrier is at the water resonance frequency.

3.3 Calibrating Pulse widths and powers: Pulses have to be calibrated accurately to suppress artifacts and maximize the signal to noise ratio $(\mathrm{S} / \mathrm{N})$ of the experiments. All pulses should be calibrated every time experiments are set up, including the pulses for ${ }^{13} \mathrm{C}$ and ${ }^{15} \mathrm{~N}$.

1. ${ }^{1} \mathbf{H}$ Square pulses: Both hard and decoupling ${ }^{1} \mathrm{H}$ square pulses are calibrated using the water signal.

a. Set the ${ }^{1} \mathrm{H}$ RF carrier on the water resonance frequency.

b. Record a series of single scan 1D experiments at the desired $B_{l}$ field strength varying the pulse width in steps of $1 \mu$ s around the pulse width for a $360^{\circ}(2 \pi)$ pulse. Process the FIDs in absolute value mode. The spectrum with the lowest water peak intensity is the one where the pulse width is closest to the $2 \pi$ pulse. Subsequently, array the pulse width in smaller steps around the pulse width estimated around this value to obtain a more accurate estimate of the pulse width. Typical array spacing for the ${ }^{1} \mathrm{H}$ hard pulses is $0.1 \mu$ s but can be larger for longer square pulses like decoupling pulses. The ${ }^{1} \mathrm{H}$ hard pulses are applied at the highest possible power and typical pulse widths for hard $90^{\circ}$ pulses are between 6.5 and $9 \mu$ s. CPMG pulses are applied at a lower power to avoid damaging the probe and pulsewidths for the refocusing $180^{\circ}$ pulses vary between 21 and $22 \mu$ s.

2. ${ }^{1}$ H Selective pulses for water suppression: Due to radiation dampening $90^{\circ}$ water selective pulses that bring water magnetization down from the $\mathrm{Z}$ axis to the transverse plane 
are longer than those that take water from the transverse plane to the $\mathrm{Z}$ axis [7]. The water selective pulses are calibrated using a two-pulse sequence where the first pulse and the second pulse are $180^{\circ}$ out of phase. To calibrate the pulse that brings the magnetization down to the transverse plane from the $\mathrm{Z}$ axis the first pulse is the water selective pulse that has to be calibrated while the second one is a hard $90^{\circ}$ proton pulse. To calibrate the pulse that takes water magnetization from the transverse plane to the $\mathrm{Z}$ axis, the order of the hard and selective pulses is reversed.

a. Place the ${ }^{1} \mathrm{H}$ RF carrier on the water resonance and set the hard pulse power and pulse width to that of a $90^{\circ}$ pulse.

b. Select the shape and set the power for the water selective pulse. Typical shapes include a square pulse, a SEDUCE pulse [13] and the EBURP pulse [14].

c. Record a series of single scan 1D experiments varying the selective pulse width around the expected pulse width. The step size can be 10 to $20 \mu$ s for 1.5 to $2 \mathrm{~ms}$ selective pulses and 50-100 $\mu$ s for 5 to $10 \mathrm{~ms}$ selective pulses. Process the FIDs in the absolute value mode. The minimum water peak intensity is obtained when the selective pulse width is equal to the length of the $90^{\circ}$ pulse. Alternatively one can array the power of the selective pulse while keeping the pulse width fixed. At $500 \mathrm{MHz}$ the $90^{\circ}$ square water selective pulses are $\sim 1.5 \mathrm{~ms}$ long, SEDUCE pulses are $\sim 1.8 \mathrm{~ms}$ and the EBURP pulse is $\sim 7 \mathrm{~ms}$. These pulses are usually not scaled with field strength.

d. Set the selective pulse width and power to the optimized values and array the small angle phase of the selective pulse around $0^{\circ}$ in steps of $1^{\circ}$. Process the spectra as above to obtain the phase at which the water intensity is the lowest. For newer spectrometers it is very close to $0^{\circ}$ but can be larger for older spectrometers mainly due to non-linearity of the amplifiers and pulse imperfections.

3. ${ }^{15} \mathrm{~N}$ square pulse: ${ }^{15} \mathrm{~N}$ square pulses are calibrated using a modified HSQC experiment that contains an extra ${ }^{15} \mathrm{~N}$ pulse that is to be calibrated placed between two $\mathrm{Z}$ gradients. This calibration element is placed after the first ${ }^{1} \mathrm{H} \rightarrow{ }^{15} \mathrm{~N}$ INEPT transfer. The first INEPT period [15] generates a density matrix element proportional to the $2 \mathrm{H}_{\mathrm{z}} \mathrm{N}_{\mathrm{z}}$ longitudinal two-spin order magnetization. Applying an extra ${ }^{15} \mathrm{~N}$ (or ${ }^{1} \mathrm{H}$ ) pulse followed by a strong $\mathrm{Z}$ gradient will destroy all the magnetization and no signal will be observed when the pulse width corresponds to $90^{\circ}$ pulse. It is best to use a sensitivity enhanced HSQC with gradient 
selection [16] for this purpose. $A{ }^{15} \mathrm{~N}$ labeled protein sample in $\mathrm{H}_{2} \mathrm{O}$ is required to perform this calibration.

a. Place the ${ }^{1} \mathrm{H}$ RF carrier on the water resonance and the ${ }^{15} \mathrm{~N}$ carrier at $119 \mathrm{ppm}$, which is in the middle of the amide region.

b. Set powers and pulse widths of the ${ }^{1} \mathrm{H}$ hard and water selective pulses. Set the small angle phase shift of the water selective pulses.

c. Set the ${ }^{15} \mathrm{~N}$ hard and decoupling pulse widths and powers to reasonable values. Set the shape and the power of the pulse to be calibrated to the desired value. These can be taken from the last calibration performed on the sample or from calibrations performed on a standard sample.

d. Set the pulse width of the ${ }^{15} \mathrm{~N}$ pulse to be calibrated to 0 and record the first increment of the HSQC experiment (no $t_{1}$ delay) with 8 scans. Process the FID with water suppression and suitable apodization and phase the spectrum focusing on the amide ${ }^{1} \mathrm{H}$ region. Ensure that water suppression is adequate and that the $\mathrm{S} / \mathrm{N}$ of the amide protons is adequate. If not one can increase the number of scans. An acquisition time of $64 \mathrm{~ms}$ and an interscan delay of $1.5-2 \mathrm{~s}$ is usually sufficient.

e. Record a series of 1D spectra as above varying the pulse width of the pulse to be calibrated. The pulse width is varied in steps of 0.33 to $0.5 \mu$ s for hard and CPMG pulses that are $\sim 35-45 \mu$ s long. Process the spectrum exactly as in the previous step. The amide peaks will be positive when the pulse width is less than the $90^{\circ}$ pulse width and negative when the pulse width is greater than the $90^{\circ}$ pulse width. Almost no signal will be seen when the pulse width is almost $90^{\circ}$. Hard ${ }^{15} \mathrm{~N} 90^{\circ}$ pulses are $\sim 35 \mu$ s long. To avoid damaging the probe CPMG pulses are applied at a lower power with $180^{\circ}$ pulsewidths ranging between 80 and 90 $\mu$ s.

4. ${ }^{15}$ N REBURP pulse: The refocusing REBURP pulse [14] can be calibrated in a manner similar to the hard pulse because when the pulse is applied for half its duration it behaves like a $90^{\circ}$ pulse with respect to starting $\mathrm{Z}$ magnetization.

a. Set the shape of the pulse to be calibrated to a REBURP pulse and the power to the desired value.

b. Record a series of 1D spectra using the first increment of the HSQC experiment as in step 3 varying the width of the REBURP pulse. The pulse width is varied in steps 1-2 $\mu$ s around the estimated $90^{\circ}$ value. For example if the desired pulse width of the REBURP pulse is 1.5 
$\mathrm{ms}$ then the pulse width is arrayed in steps of $\sim 2 \mu$ s around $0.75 \mathrm{~ms}$. Process and compare the series of 1D spectra as in step 3 to obtain the $90^{\circ}$ pulse width. Doubling this value will give the pulse width of the REBURP.

c. Confirm that the REBURP pulse is accurately calibrated. Set the pulse width of the REBURP to the calibrated value. Record two spectra, one with the REBURP pulse power set to 0 and one with the REBURP pulse power set to the desired value. When the calibration is accurate the amide region will be inverted in the second spectrum, with the height of each amide peak in the second spectrum being at least $95 \%$ of its height in the first spectrum. The amide selective ${ }^{15} \mathrm{~N}$ REBURP pulse width is typically $\sim 1.4 \mathrm{~ms}$ (at $16.65 \mathrm{~T}$ ) and needs to be scaled according to field strength.

5. ${ }^{1}$ H REBURP pulse: The ${ }^{1} \mathrm{H}$ REBURP pulse can be calibrated in a manner similar to the ${ }^{15} \mathrm{~N}$ REBURP pulse. The ${ }^{15} \mathrm{~N}$ REBURP pulse is replaced by the ${ }^{1} \mathrm{H}$ REBURP pulse in the above sequence and the process is repeated with two small differences. i) During the ${ }^{1} \mathrm{H}$ REBURP pulse the ${ }^{1} \mathrm{H}$ carrier is placed at $\sim 7.7 \mathrm{ppm}$ in the middle of the amide proton region. ii) A water selective pulse that is usually applied after the ${ }^{1} \mathrm{H}$ to ${ }^{15} \mathrm{~N}$ INEPT period is turned off. The amide selective ${ }^{1} \mathrm{H}$ REBURP pulse is generally chosen to be $\sim 1.4 \mathrm{~ms}$ long (at $16.65 \mathrm{~T}$ ) and needs to be scaled according to the field strength.

6. ${ }^{13} \mathrm{C}$ Square/Shaped pulses via HNCO experiments: In uniformly ${ }^{15} \mathrm{~N} /{ }^{13} \mathrm{C}$ enriched protein samples dissolved in water the ${ }^{13} \mathrm{C}$ pulses are calibrated in a manner similar to the ${ }^{15} \mathrm{~N}$ pulses. Here the first scan of a gradient selected sensitivity enhanced HNCO experiment [17] is used instead of the HSQC experiment. A Z gradient followed by the extra ${ }^{13} \mathrm{C}$ pulse to be calibrated followed by a strong $\mathrm{Z}$ gradient is inserted after the ${ }^{15} \mathrm{~N} \rightarrow{ }^{13} \mathrm{CO}$ INEPT period and calibrated in a manner identical to the ${ }^{15} \mathrm{~N}$ pulses as described above.

7. ${ }^{13} \mathrm{C}$ Square/Shaped pulse via HSQC experiments: ${ }^{13} \mathrm{C}$ pulses can be calibrated using ${ }^{13} \mathrm{C}$ labeled methyl groups in samples which are not dissolved in water or when the carbonyl groups are not ${ }^{13} \mathrm{C}$ enriched. As in the case of the ${ }^{15} \mathrm{~N}$ pulse calibration an extra ${ }^{13} \mathrm{C}$ pulse to be calibrated followed by a strong gradient is inserted after the ${ }^{1} \mathrm{H} \rightarrow{ }^{13} \mathrm{C}$ INEPT in a standard ${ }^{1} \mathrm{H}-{ }^{13} \mathrm{C}$ HSQC experiment. The procedure for calibration is similar to that for the ${ }^{15} \mathrm{~N}$ pulse. The ${ }^{13} \mathrm{C}$ carrier is placed in the middle of methyl region $(\sim 20.5 \mathrm{ppm})$ and the pulse width is 
calibrated by looking at methyl proton peak intensities between -0.5 and $1 \mathrm{ppm}$ just as one focused on the amide protons during the ${ }^{15} \mathrm{~N}$ calibration as described above.

3.4 Measuring Sample heating: Experiments with ${ }^{1} \mathrm{H}$ decoupling can heat the samples and consequently change the kinetics of the process being studied. Hence the effect of the experiment on the sample temperature has to be measured and compensated by adjusting the VT setting (See Note 2).

1. Record a high-resolution 1D WATERGATE spectrum [7, 18] of the amide or methyl protons at $1{ }^{\circ} \mathrm{C}$ higher than the desired temperature. Methyl protons are preferred but amide protons are used when methyl protons are absent due to ${ }^{2} \mathrm{H}$ labeling.

2. Record a 1D WATERGATE spectrum of the amide or methyl protons at the desired temperature. The peaks in this spectrum will move compared to the one recorded in the previous step allowing one to calibrate peak position change as a function of temperature.

3. Using the same VT temperature, perform the CPMG experiment of interest for about five to ten minutes so that the sample temperature has reached a steady state.

4. Queue a single scan 1D WATERGATE experiment of the amide/methyl region to run immediately after the CPMG experiment from step 3. has finished recording. This experiment should have no dummy scans.

5. The peak positions would have changed compared to the spectrum recorded in step 2 due to sample heating during the CPMG experiment. By comparing the peak positions in the three spectra recorded in steps 1,2 and 4 the heating caused by the CPMG experiment can be estimated according to $\frac{\varpi_{4}-\varpi_{2}}{\varpi_{1}-\varpi_{2}}$, where $\varpi_{1}, \varpi_{2}$ and $\varpi_{4}$ are the resonance frequencies of a particular amide/methyl proton in spectra recorded in steps 1,2 and 4 respectively. Do this for 3 to 5 peaks that move consistently and take the average value.

6. The VT value can now be reduced to compensate for the heating.

3.5 CT ${ }^{15} \mathrm{~N}$ TROSY CPMG experiment: The CT ${ }^{15} \mathrm{~N}$ TROSY CPMG experiment exploits both the improved relaxation properties of the ${ }^{15} \mathrm{~N}$ TROSY component during the $T_{C P M G}$ period and the enhanced resolution of the ${ }^{15} \mathrm{~N}-{ }^{1} \mathrm{H}$ TROSY correlation map. This experiment is necessary to perform ${ }^{15} \mathrm{~N}$ CPMG experiments with larger proteins but can also be used with smaller proteins. This experiment has to be performed with uniformly ${ }^{15} \mathrm{~N}$ enriched samples. Uniform ${ }^{2} \mathrm{H}$ labeling will improve the performance of the experiment but is not required. If 
the sample is ${ }^{13} \mathrm{C}$ enriched a single carbon pulse is used to refocus the ${ }^{13} \mathrm{C}-{ }^{15} \mathrm{~N}$ scalar couplings during the ${ }^{15} \mathrm{~N}$ chemical shift evolution.

1. Place the ${ }^{1} \mathrm{H}$ RF carrier on the water resonance, the ${ }^{15} \mathrm{~N}$ carrier at $119 \mathrm{ppm}$ in the middle of the amide region and the ${ }^{13} \mathrm{C}$ carrier at $118 \mathrm{ppm}$ in between ${ }^{13} \mathrm{CO}$ and ${ }^{13} \mathrm{C} \alpha$ resonances. Values of the delays are available in [19].

2. Set powers and pulse widths of the ${ }^{1} \mathrm{H}$ hard and water selective $90^{\circ}$ pulses.

3. Set powers and pulse widths of the ${ }^{15} \mathrm{~N} 90^{\circ}$ hard pulses and CPMG $\pi$ pulses (see Note 3). To avoid damage to the probe, CPMG pulses are applied at lower power with the $180^{\circ}$ pulse width ranging from 80 to $90 \mu \mathrm{s}$. A REBURP $\pi$ pulse can be applied during the first INEPT period to suppress resonances arising from lysine and arginine sidechains.

4. If the sample is ${ }^{13} \mathrm{C}$ enriched set the power for a $\sim 00 \mu$ s adiabatic pulse [20] with adequate bandwidth to invert both the carbonyls and alpha carbons.

5. Set the $T_{C P M G}$ delay (See Note 4). $T_{C P M G}$ values usually lie between 20 and $40 \mathrm{~ms}$. Values above $40 \mathrm{~ms}$ are not chosen as applying a large number of ${ }^{15} \mathrm{~N}$ refocusing pulses can damage the probe.

6. Set the number of scans in multiples of the phase cycle to obtain good signal to noise. Typical values are 8 or 16 scans.

7. Decide on the $v_{\mathrm{CPMG}}$ values to use. $v_{\mathrm{CPMG}}$ values that can be used are $\frac{n}{T_{C P M G}}$ where $\mathrm{n}$ is an integer starting from $1[19,21]$. $v_{\mathrm{CPMG}}$ values up to $\sim 1000 \mathrm{~Hz}$ can be used with current probes. Experiments are performed for $\sim 15$ to $20 v_{\mathrm{CPMG}}$ values ranging from $\frac{1}{T_{C P M G}}$ to $1000 \mathrm{~Hz}$ with two or three repeat values for error estimation. Lower $v_{\text {CPMG }}$ values are sampled more closely. 8. Record ${ }^{1} \mathrm{H}^{-15} \mathrm{~N}$ correlation maps for each of the $v_{\mathrm{CPMG}}$ values and a reference plane without the $T_{C P M G}$ delay in an interleaved manner. The usual experimental time varies from 12 to 24 hours. See Note 5.

3.6 CT ${ }^{15} \mathbf{N}$ CPMG experiment with ${ }^{1} \mathbf{H}$ decoupling: Here the amide proton is decoupled from the amide ${ }^{15} \mathrm{~N}$ nucleus using ${ }^{1} \mathrm{H}$ CW decoupling during the $T_{C P M G}$ delay [21]. This experiment is particularly useful while performing CPMG experiments in alignment media and is also significantly less prone to off-resonance artifacts. If the sample is ${ }^{13} \mathrm{C}$ enriched, a single carbon pulse is used to refocus the ${ }^{13} \mathrm{C}-{ }^{15} \mathrm{~N}$ couplings during ${ }^{15} \mathrm{~N}$ evolution.

1. Set the ${ }^{1} \mathrm{H},{ }^{15} \mathrm{~N}$ and ${ }^{13} \mathrm{C}$ carrier offsets as described in section 3.5, step 1 . The ${ }^{1} \mathrm{H}$ carrier is moved to $8.1 \mathrm{ppm}$ in the middle of the amide region when $\mathrm{CW}{ }^{1} \mathrm{H}$ decoupling is performed. Values of delays are available in [21]. 
2. Set powers and pulse widths of the ${ }^{1} \mathrm{H}$ hard, water selective $90^{\circ}$ pulses and $\mathrm{CW}$ decoupling field. An EBURP1 [21] pulse is used to bring the water magnetization from the $\mathrm{Z}$ axis to the transverse plane. The $\mathrm{CW}{ }^{1} \mathrm{H}$ decoupling is applied at $15 \mathrm{kHz}$ at $18.8 \mathrm{~T}[21,22]$ and scaled according to the field strength.

3. Set powers and pulse widths for the ${ }^{15} \mathrm{~N}$ pulses as in step 3 of section 3.5. Additionally set the power for ${ }^{15} \mathrm{~N}$ decoupling during acquisition. At $18.8 \mathrm{~T}$ WALTZ-16 ${ }^{15} \mathrm{~N}$ decoupling is performed with $B_{1}$ of $1.3 \mathrm{kHz}$ [23]. This ${ }^{15} \mathrm{~N}$ decoupling field should be scaled with magnetic field strength.

4. If the sample is ${ }^{13} \mathrm{C}$ enriched use a adiabatic pulse for decoupling as described in section 3.5 , step 4 .

5. Set the $T_{C P M G}$ delay as described in section 3.5, step 5. $T_{C P M G}$ values usually lie between 20 and $30 \mathrm{~ms}$. Due to heating effects of the ${ }^{1} \mathrm{H} \mathrm{CW}$ field it is prudent to set $T_{C P M G} \leq 30 \mathrm{~ms}$.

6. Measure the heating due to the ${ }^{1} \mathrm{H} \mathrm{CW}$ field and compensate for it by adjusting the VT setting. Alternatively see Note 2.

7. Set the number scans, choose the $v_{\mathrm{CPMG}}$ values and run the experiment as described in steps 6,7 and 8 of section 3.5 .

3.7 Amide ${ }^{1} \mathbf{H}$ CPMG experiment: The amide ${ }^{1} \mathrm{H}$ CPMG experiment is used to study dynamics at the amide proton sites. A uniformly ${ }^{15} \mathrm{~N}$ labeled protein sample is used. ${ }^{2} \mathrm{H}$ enrichment is desirable but not necessary. To suppress peaks arising from ROE transfer the experiment is performed on $2 \mathrm{H}_{\mathrm{x}} \mathrm{N}_{\mathrm{z}}$ antiphase magnetization. If the sample is ${ }^{13} \mathrm{C}$ enriched, a single carbon pulse is used to refocus the ${ }^{13} \mathrm{C}-{ }^{15} \mathrm{~N}$ couplings during ${ }^{15} \mathrm{~N}$ evolution. Details can be found in $[24,25]$.

1. Set the ${ }^{1} \mathrm{H},{ }^{15} \mathrm{~N}$ and ${ }^{13} \mathrm{C}$ carrier offsets as described in section 3.5, step 1 . The ${ }^{1} \mathrm{H}$ carrier is moved to $8.1 \mathrm{ppm}$ in the middle of the amide region during the CPMG period. Values of delays are available in [24, 25]. The modifications described in [25] have to be used to if the procedure described here is to be used.

2. Set powers and pulse widths of the ${ }^{1} \mathrm{H}$ hard, $\mathrm{CPMG}$ and water selective $90^{\circ}$ pulses. To avoid damaging the probe the ${ }^{1} \mathrm{H}$ CPMG pulse is applied at a lower power with a $180^{\circ}$ pulsewidth of $\sim 21 \mu \mathrm{s}$. A REBURP pulse is used to invert the amide protons without perturbing the $\alpha$ protons in the middle of the CPMG period. The length of the REBURP and the amide carrier frequency need to be optimized to cover as many if not all the amides. A 
square $180^{\circ}$ pulse applied at the same power as the other CPMG pulses can be used if the sample is labeled with ${ }^{2} \mathrm{H}$ at the $\mathrm{H}^{\alpha}$ positions.

3. Set powers and pulse widths of the ${ }^{15} \mathrm{~N}$ pulses as described in section 3.6, step 3 .

4. If the sample is ${ }^{13} \mathrm{C}$ enriched use a adiabatic pulse for decoupling as described in section 3.5 , step 4.

5. Set the $T_{C P M G}$ delay and number of scans as described in section 3.5 , steps $5 \& 6 . T_{C P M G}$ values usually lie between 20 and $30 \mathrm{~ms}$.

6. Decide on the $v_{\text {CPMG }}$ values to use and run the experiment as described steps 7 and 8 of section 3.5. Unlike ${ }^{15} \mathrm{~N}$ CPMG experiments $v_{\mathrm{CPMG}}$ values up to $2000 \mathrm{~Hz}$ can be used with current probes. Experiments are performed for $\sim 15-25 v_{\mathrm{CPMG}}$ values ranging from $\frac{1}{T_{C P M G}}$ to $2000 \mathrm{~Hz}$ with two or three repeat values for error estimation.

3.8 ${ }^{13}$ CO CPMG experiment: The carbonyl CPMG experiment is performed using a uniformly ${ }^{15} \mathrm{~N} /{ }^{13} \mathrm{C}$ enriched sample.

1. Place the ${ }^{1} \mathrm{H}$ carrier on the water resonance, the ${ }^{13} \mathrm{C}$ carrier at $176 \mathrm{ppm}$ in the middle of the carbonyl region and the ${ }^{15} \mathrm{~N}$ carrier at $119 \mathrm{ppm}$ in the middle of the amide region. Values of delays are available in [26].

2. Set powers and pulse widths of the ${ }^{1} \mathrm{H}$ hard and water selective $90^{\circ}$ pulses as described in step 2 of section 3.5. Additionally here WALTZ-16 decoupling is carried out and is performed at $6 \mathrm{kHZ}$ at $18.8 \mathrm{~T}$.

3. Set powers and pulse widths of the ${ }^{15} \mathrm{~N} 90^{\circ}$ hard pulses as in step 3 of section 3.5 and power for the ${ }^{15} \mathrm{~N}$ decoupling sequence as in step 3 of section 3.6.

4. Square ${ }^{13} \mathrm{C} 90^{\circ}$ pulses are applied at a field strength (given in $\mathrm{Hz}$ ) of $\Delta \Omega / \sqrt{ } 15$ where $\Delta \Omega$ is $118 \mathrm{ppm} \times \mathrm{dfrq}$ where dfrq is the carbon frequency in $\mathrm{MHz}$. The square ${ }^{13} \mathrm{C} 180^{\circ}$ pulse is applied at the highest possible power leading to a pulse width of $\sim 25 \mu \mathrm{s}(20 \mathrm{kHz})$ for the $180^{\circ}$ pulse. The SEDUCE-1 sequence [13] is used to decouple the ${ }^{13} \mathrm{C} \alpha$ from ${ }^{13} \mathrm{CO}$ during the $\mathrm{t}_{1}$ ${ }^{13} \mathrm{CO}$ chemical shift evolution period. The SEDUCE-1 pulses are cosine modulated at a frequency of $118 \mathrm{ppm}$ with a maximum $B_{1}$ of $3.14 \mathrm{kHz}$ at $11.7 \mathrm{~T}$. The length of the SEDUCE- $190^{\circ}$ pulse is $\sim 335 \mu$ s at $11.7 \mathrm{~T}$ and is scaled according to the field strength. Note that due to the cosine modulation decoupling is applied at four times the power. During acquisition the ${ }^{1} \mathrm{HN}-{ }^{13} \mathrm{CO} \mathrm{J}$ coupling is decoupled using the WURST-2 decoupling sequence with a bandwidth of $12 \mathrm{ppm}$ [20]. A maximum $B_{l}$ of $0.6 \mathrm{kHz}$ is required at $18.85 \mathrm{~T}$ and should be scaled with field strength. A shaped pulse [26] related to the REBURP pulse is 
used during the ${ }^{13} \mathrm{C}$ CPMG period. The length of this $180^{\circ}$ pulse is $380 \mu$ s at $18.85 \mathrm{~T}$ and should be scaled according the magnetic field strength. This pulse is calibrated in a manner similar to the REBURP.

5. Set the $T_{C P M G}$ delay and number of scans according to steps 5 and 6 of section 3.5. $T_{C P M G}$ values usually lie between 20 and $30 \mathrm{~ms}$.

6. Decide on the $v_{\text {CPMG }}$ values to use as described in step 7 of section 3.5 keeping in mind that although $v_{\mathrm{CPMG}}$ values up to $1000 \mathrm{~Hz}$ can be used with current probes, at lower magnetc field strengths the length of the shaped ${ }^{13} \mathrm{C} C \mathrm{CPMG}$ pulse places an upper limit on the highest $v_{\mathrm{CPMG}}$ value that can be used. Experiments are performed for $\sim 15 v_{\mathrm{CPMG}}$ values.

3.9 ${ }^{1} \mathrm{H} \boldsymbol{\alpha}$ CPMG experiment: The ${ }^{1} \mathrm{H} \alpha$ chemical shift provides useful structural information and is extremely useful for structure determination of the minor state. However the $\mathrm{H} \alpha$ nucleus is scalar coupled to amide protons (HN) and $\mathrm{H} \beta$ nuclei. The scalar coupling to the amide protons can be eliminated by dissolving the sample in $\mathrm{D}_{2} \mathrm{O}$, so that the amide proton is replaced by a ${ }^{2} \mathrm{H}$ nucleus. However there is no straightforward way to produce protein molecules that are $\mathrm{H} \alpha$ labeled while being $\sim 100 \%{ }^{2} \mathrm{H}$ enriched at the $\mathrm{H} \beta$ position. By overexpressing the protein in $E$ coli grown in $50 \% \mathrm{D}_{2} \mathrm{O}$ minimal media with ${ }^{2} \mathrm{H} /{ }^{13} \mathrm{C}$ glucose as the sole carbon source, the $\mathrm{H} \beta$ is $50-88 \%$ deuterated reducing the effect of ${ }^{3} \mathbf{J}_{\mathrm{H \alpha H} \beta}$ coupling. The ${ }^{1} \mathrm{H} \alpha \mathrm{CPMG}$ experiments consist of a $\mathrm{J}$ refocusing element in the middle of the CPMG period [27]. Neglecting relaxation, this $\mathbf{J}$ refocusing element uses the ${ }^{1} \mathbf{J}_{\mathrm{C} \alpha \mathrm{H} \alpha}$ coupling to refocus the effects of the ${ }^{3} \mathrm{~J}_{\mathrm{HaH} \beta}$ coupling to first order. In this experiment dispersion profiles are obtained by analyzing ${ }^{1} \mathrm{H}^{-13} \mathrm{C} \alpha$ correlation maps.

1. Place the ${ }^{1} \mathrm{H}$ RF carrier on the water resonance, the ${ }^{13} \mathrm{C}$ carrier at $58 \mathrm{ppm}$ in the $\mathrm{C} \alpha$ region and the ${ }^{15} \mathrm{~N}$ carrier at $119 \mathrm{ppm}$ in the amide region. Values of delays are available in [27].

2. Set powers and pulse widths of the ${ }^{1} \mathrm{H}$ hard and CPMG pulses. CPMG pulses are applied at a lower power as described in section 3.7, step 2 .

3. Set the power and pulse width for the ${ }^{15} \mathrm{~N}$ hard pulse if the sample is ${ }^{15} \mathrm{~N}$ enriched. As there is no water in the sample the ${ }^{15} \mathrm{~N}$ pulse width and power have to be set to the last values calibrated on the same sample.

4. Set the power and pulse width for the ${ }^{13} \mathrm{C}$ hard pulse and decoupling sequences. During acquisition ${ }^{13} \mathrm{C}$ decoupling is performed using a $2.5 \mathrm{kHz}$ WALTZ-16 decoupling scheme at $18.8 \mathrm{~T}$. The decoupling power has to be scaled with field strength. Carbonyl and ${ }^{13} \mathrm{C} \beta$ are 
decoupled from ${ }^{13} \mathrm{C} \alpha$ during $\mathrm{t}_{1}$ evolution. ${ }^{13} \mathrm{CO}$ decoupling is performed using a CAWURST8 scheme $[28,29]$ with $10 \mathrm{ppm}$ bandwidth centered at $175 \mathrm{ppm}$ and applied with a maximum field strength of $0.4 \mathrm{kHz}$ at $18.8 \mathrm{~T} .{ }^{13} \mathrm{C} \beta$ decoupling is performed using i) A CAWURST-8 scheme with a $30 \mathrm{ppm}$ bandwidth swept from 15 to $45 \mathrm{ppm}$ centered at $30 \mathrm{ppm}$ with a maximum $\mathrm{B}_{1}$ of $0.5 \mathrm{kHz}$ at $18.8 \mathrm{~T}$ and ii) A second CAWURST-8 scheme with a $30 \mathrm{ppm}$ bandwidth swept from 101 to $71 \mathrm{ppm}$ centered at $86 \mathrm{ppm}$ with a maximum $\mathrm{B}_{1}$ of $0.5 \mathrm{kHz}$ at 18.8 $\mathrm{T}$. The second field eliminates decoupling artifacts. During the the $\mathrm{J}$ refocusing element $\mathrm{C} \beta$ decoupling is performed using a WURST-2 scheme [30] centered at $27.5 \mathrm{ppm}$ with a 25 ppm bandwidth and a maximum $B_{l}$ of $1.22 \mathrm{kHz}$ at $18.8 \mathrm{~T}$.

5. Set the $T_{C P M G}$ delay (See Note 4). $T_{C P M G}$ values can be quite low due to the short $\mathrm{H} \alpha T_{2}$ values and usually lie between 15 and $30 \mathrm{~ms}$.

6. Set the number scans in multiples of the phase cycle to obtain good signal to noise. The sensitivity of these experiments is low due to $50 \%{ }^{1} \mathrm{H}$ labeling at the $\alpha$ position and because the experiments are not carried out in a sensitivity-enhanced manner. Hence significantly more number of scans are required with typical values ranging from 24 to 48 scans.

7. Decide on the $v_{\mathrm{CPMG}}$ values to use. $v_{\mathrm{CPMG}}$ values that can be used are $\frac{2 n}{T_{C P M G}}$ where $\mathrm{n}$ is an integer starting from 1. $v_{\mathrm{CPMG}}$ values upto $1000 \mathrm{~Hz}$ can be used. Experiments are performed for $\sim 10$ to $15 v_{\mathrm{CPMG}}$ values ranging from $\frac{2}{T_{C P M G}}$ to $1000 \mathrm{~Hz}$ with two or three repeat values for error estimation. Lower $v_{\mathrm{CPMG}}$ values are sampled more closely.

8. Record ${ }^{1} \mathrm{H}-{ }^{13} \mathrm{C}$ correlation maps for each of the $v_{\mathrm{CPMG}}$ values and a reference plane without the $T_{C P M G}$ delay in an interleaved manner. The usual experimental time varies from 24 to 48 hours (See Note 5).

3.10 ${ }^{13} \mathrm{C} \alpha$ CPMG experiment: The ${ }^{13} \mathrm{C} \alpha$ chemical shift provides very valuable structural information. As the $\mathrm{C} \alpha$ chemical shifts are not well separated from the $\mathrm{C} \beta$ chemical shifts a sample that is ${ }^{13} \mathrm{C}$ enriched at only the $\mathrm{C} \alpha$ sites is used. In this experiment dispersion profiles are obtained by analyzing ${ }^{1} \mathrm{H}-{ }^{13} \mathrm{C} \alpha$ correlation maps.

1. Place the ${ }^{1} \mathrm{H}$ RF carrier on the water resonance, the ${ }^{13} \mathrm{C}$ carrier at $55 \mathrm{ppm}$ in the $\mathrm{C} \alpha$ region and the ${ }^{15} \mathrm{~N}$ carrier at $119 \mathrm{ppm}$ in the middle of the amide region. Values of delays are available in [11].

2. Set powers and pulse widths of the ${ }^{1} \mathrm{H}$ hard pulses. The ${ }^{1} \mathrm{H} \mathrm{CW}$ decoupling field during the CPMG period is applied at a $B_{l}$ field strength of $\sim 14.5 \mathrm{kHz}$. Purge trim CW pulses to dephase water are applied at $16 \mathrm{kHz}$. Proton to carbon and carbon to proton transfers are carried out 
using DIPSI-2 sequences with ${ }^{1} \mathrm{H}$ and ${ }^{13} \mathrm{C}$ fields of $8 \mathrm{kHz}$ at $18.8 \mathrm{~T}$. ${ }^{1} \mathrm{H}$ decoupling during ${ }^{13} \mathrm{C}$ $\mathrm{t}_{1}$ evolution is performed using a $5 \mathrm{kHz}(18.8 \mathrm{~T}) \mathrm{WALTZ}-16$ sequence.

3. Set the power and pulse width for the ${ }^{15} \mathrm{~N}$ hard pulse.

4. Set power and pulse width for the ${ }^{13} \mathrm{C}$ hard pulse and CPMG pulses. The CPMG pulses are applied at lower field strength with a $180^{\circ}$ pulsewidth of $\sim 31 \mu \mathrm{s} .{ }^{13} \mathrm{C}$ decoupling during acquisitions is performed using a $2.5 \mathrm{kHz}(18.8 \mathrm{~T})$ WALTZ-16 sequence. The decoupling field strength is scaled with the spectrometer frequency. Proton to carbon and carbon to proton transfers are carried out using the DIPSI-2 sequence [31] with proton and carbon fields of $(8 \mathrm{kHz}$ at $18.8 \mathrm{~T})$. The decoupling field strength is scaled with magnetic field strength.

5. Set the $T_{C P M G}$ delay (See Note 4). $T_{C P M G}$ values can be quite low due to the short $\mathrm{C} \alpha{ }^{\mathrm{T} 2}$ values and usually lie between 15 and $30 \mathrm{~ms}$. Further due to heating effects of the ${ }^{1} \mathrm{H} \mathrm{CW}$ field it is prudent to set $T_{C P M G} \leq 30 \mathrm{~ms}$.

6. Measure the heating due to the ${ }^{1} \mathrm{H} \mathrm{CW}$ field and compensate for it by reducing the VT setting. Alternatively see Note 2.

7. Set the number of scans in multiples of the phase cycle to obtain good $\mathrm{S} / \mathrm{N}$ ratio. Due to the method of sample production the ${ }^{13} \mathrm{C} \alpha$ enrichment is at most $50 \%$. Further the experiment is not carried out in a sensitivity-enhanced manner. Hence a significantly larger number of scans are required with typical values ranging between 16 to 48 scans.

8. Decide on the $v_{\mathrm{CPMG}}$ values as described in step 7 of section 3.5. Note that although $v_{\mathrm{CPMG}}$ values upto $1500 \mathrm{~Hz}$ can be used with current probes values up to $1000 \mathrm{~Hz}$ are preferred.

9. Run the experiment as described in step 8 of section 3.9.

3.11 Amide ${ }^{1} \mathrm{H}^{15} \mathrm{~N}$ ZQ/DQ CPMG experiment: In addition to ${ }^{1} \mathrm{H}$ and ${ }^{15} \mathrm{~N}$ single quantum transitions one can also excite zero quantum (ZQ) and double quantum (DQ) transitions in the coupled amide ${ }^{1} \mathrm{H} /{ }^{15} \mathrm{~N}$ spin system. Relaxation dispersion curves for the ZQ and DQ transitions can also be recorded using CPMG experiments [32]. During ZQ/DQ CPMG experiments, $\mathrm{ZQ} / \mathrm{DQ}$ coherence is generated and refocusing pulses are applied simultaneously on both the ${ }^{15} \mathrm{~N}$ and ${ }^{1} \mathrm{H}$ nuclei during the $T_{C P M G}$ period. In the case of exchange the change in the frequency of the ZQ transition between the minor and major states in ppm units is $\left|\Delta \varpi_{N^{-}} \Delta \varpi_{H}\right|$ while the change in the frequency of the DQ transition is $\left|\Delta \varpi_{N}+\Delta \varpi_{H}\right|$. Hence if $\left|\Delta \varpi_{N}\right|>0$ and $\left|\Delta \varpi_{H}\right|>0$ then either the ZQ or the DQ transitions will give larger dispersions than the SQ experiments making them more sensitive to the underlying exchange 
process in CPMG experiments. Additionally, ZQ/DQ CPMG experiments also provide information about the relative signs of $\Delta \varpi_{\mathrm{N}}$ and $\Delta \varpi_{\mathrm{H}}$. If the size of the DQ dispersion is larger than the ZQ dispersion then $\Delta \varpi_{\mathrm{N}}$ and $\Delta \varpi_{\mathrm{H}}$ have the same sign and the opposite is true when $\Delta \varpi_{\mathrm{N}}$ and $\Delta \varpi_{\mathrm{H}}$ have opposite signs. Relaxation dispersion curves are obtained by analyzing ${ }^{1} \mathrm{H}-{ }^{15} \mathrm{~N}$ correlation maps and the experiment is performed with ${ }^{15} \mathrm{~N} /{ }^{2} \mathrm{H}$ enriched proteins dissolved in $90 \% \mathrm{H}_{2} \mathrm{O} / 10 \% \mathrm{D}_{2} \mathrm{O}$ to back exchange the amide protons.

1. Set the ${ }^{1} \mathrm{H}$ and ${ }^{15} \mathrm{~N}$ carrier frequencies as described in step 1 of section 3.5. Values of delays are available in [32].

2. Set powers and pulse width of the ${ }^{1} \mathrm{H}$ hard, $\mathrm{CPMG}$ pulses and water selective pulses as described in step 2 of section 3.7. A water selective EPURP1 pulse is used to bring the water magnetization from the $\mathrm{Z}$ axis to the transverse plane.

3. Set powers and pulse widths of ${ }^{15} \mathrm{~N}$ hard and CPMG pulses as described in step 3 of section 3.5. Here also magnetization is transferred to the TROSY component of ${ }^{1} \mathrm{H}$ magnetization at the end of the pulse sequence and data is acquired without ${ }^{15} \mathrm{~N}$ decoupling.

4. Set the $T_{C P M G}$ delay as in step 5 of section 3.5 and check the $\mathrm{S} / \mathrm{N}$ in both the ZQ and DQ experiments. It is best to use the same $T_{C P M G}$ delay for both.

5. Set the number scans in multiples of the phase cycle to obtain good signal to noise. Typical values are 16 or 24 scans.

6. Decide on the $v_{\mathrm{CPMG}}$ values to use as described in step 7 of section 3.5. $v_{\mathrm{CPMG}}$ values that can be used are $\frac{2 n}{T_{C P M G}}$ where $\mathrm{n}$ is an integer starting from 1. $v_{\mathrm{CPMG}}$ values up to $1000 \mathrm{~Hz}$ can be used with current probes.

7. Record ${ }^{1} \mathrm{H}^{-15} \mathrm{~N}$ correlation maps for each of the $v_{\mathrm{CPMG}}$ values and a reference plane without the $T_{C P M G}$ delay for both the ZQ and DQ components in an interleaved manner. The usual experimental time varies from 24-48 hours (See Note 5).

3.12 Extracting Relaxation Dispersion Curves from the data: The NMR data is typically processed using standard programs like NMRPipe [33] and visualized using programs like SPARKY [34]. Peaks intensities in the different 2D planes can be extracted using programs like FUDA [35], PINT [36] and MUNIN [37]. In all three of these programs one can impose that only the peak intensity varies from plane to plane while the line shape and peak position remain the same in the all planes reducing the number of fitting parameters and improving the precision of the extracted intensities. The programs are also capable of dealing with partially overlapped peaks. The quality of fits can be gauged in FUDA and PINT by 
visualizing the spectra along with the fitted peaks to decide if the fit is reliable. Once the peaks have been fit to the spectra the dispersion data can be calculated from the intensities. Errors in the $R_{2, e f f}$ values are estimated on the basis of repeat measurements. Generally, a minimum error between 0.3 and $0.5 \mathrm{~s}^{-1}$ is assumed to account for various systematic errors.

3.13 Analyzing Relaxation Dispersion Curves: Initially amide ${ }^{15} \mathrm{~N} /{ }^{1} \mathrm{H}$ dispersion data is recorded and analyzed. Quite often complete kinetic studies can be performed with only amide ${ }^{15} \mathrm{~N} /{ }^{1} \mathrm{H}$ dispersion data [38]. In the first step larger dispersions where the change in the $R_{2, \text { eff }}$ is greater than 5 or $10 \mathrm{~s}^{-1}$ are analyzed. A two-state exchange model is fit to the dispersion data collected at two different field strengths in a site-specific manner. The programs CATIA [39] and CHEMEX [40] perform the fits by numerically propagating the Bloch-McConnell equations [41]. From the quality of the fits and the site-specific distribution of the exchange rate $k_{e x}$ and the minor state population $p_{b}$ one can use various statistical tools to judge if there is a single global two state process or there are multiple processes. If there is a single global process then similar $k_{e x}$ and $p_{b}$ values will be obtained from almost all the sitespecific fits [42] and one can proceed to fit a single process to all the data. If the site-specific $k_{e x}$ and $p_{b}$ values differ from site to site one can consider fitting a global three state model [43] or look to see if the $k_{e x}$ and $p_{b}$ values cluster and that residues close on the structure have similar $k_{e x}$ and $p_{b}$ in which case one can fit multiple two state models to sets of residues [44]. If the exchange parameters are not ideal for a CPMG investigation one can either change the sample conditions like temperature or use other NMR experiments like CEST to study slower processes [45, 46] or $R_{l \varrho}$ experiments to study faster process [47]. If $k_{e x}$ is fast and $p_{b}$ is poorly defined one can use the shifts in peak positions between HSQC and HMQC spectra to define $p_{b}$ [25]. After the experimental conditions have been established using ${ }^{15} \mathrm{~N} /{ }^{1} \mathrm{H}$ data other ${ }^{13} \mathrm{C} /{ }^{1} \mathrm{H}$ CPMG experiments can be performed. Analysis of the relaxation dispersion data provides only the absolute value of the change in chemical shift between the ground and excited state. The sign information can be obtained comparing peak positions in HSQC spectra recorded at different fields or between HSQC and HMQC spectra recorded at the same field [48]. $R_{1 \varrho}$ experiments can also provide sign information [49]. Once the sign information is available the chemical shifts of the minor state resonances can be calculated and the spectrum of the minor state can be reconstructed.

3.14 Atomic resolution structure of the minor state: By comparing the minor state chemical shifts with the unfolded state chemical shifts [50] parts of the molecule that are 
unfolded in the minor state can be identified [51]. In fact, identification of local or complete unfolding can be done with reasonable confidence using just ${ }^{1} \mathrm{H} /{ }^{15} \mathrm{~N}$ data. A preliminary analysis of the minor state backbone chemical shifts using programs like TALOS $[52,53]$ can provide information about changes in secondary structure between the major and minor states [54-57]. In favorable cases it is possible to obtain the three-dimensional structures of proteins from just chemical shifts $[58,59]$ and the structure of a minor state of a protein has been obtained by using this strategy [55]. Using the minor state secondary or three-dimensional structural information mutants can be designed to shift the population towards the minor state conformation and test if the CPMG derived parameters are meaningful [55]. The quality of structures can significantly be improved by using residual dipolar couplings (RDCs) and residual chemical shift anisotropy shifts (RCSAs) [60, 61]. Minor state RDCs and RCSA shifts can be obtained by performing CPMG experiments in the presence of alignment media $[19,62,63]$ and these restraints can also be used to obtain structures of the minor state [54, 56, 57]. Here we have described CPMG experiments to study the dynamics at protein backbone sites. CPMG experiments have also been developed to study dynamics at sidechain sites $[8,64,65]$ and provide information regarding the conformation of the sidechains in the minor state $[66,67]$.

\section{Notes}

1. Using a thermocouple is more convenient as it does not require shimming and recording spectra to calculate the temperature like in the case of the standard sample.

2. Alternatively, some newer NMR spectrometers are equipped with a so-called NMR thermometer $[68,69]$, which measures the temperature inside the NMR sample during the experiment using a second locking agent. If such a system is available and if a second locking agent can be added without interfering with the chemical exchange process under investigation, then the NMR thermometer provides a convenient way of keeping the temperature constant during all experiments.

3. ${ }^{15} \mathrm{~N}$ pulsewidths can change when a CPMG pulse train is applied particularly when ${ }^{15} \mathrm{~N}$ CPMG experiments are performed using a cryogenically cooled probe. The problem does not occur with ${ }^{1} \mathrm{H}$ or ${ }^{13} \mathrm{C}$ CPMG experiments. To test if there is a change in the ${ }^{15} \mathrm{~N}$ pulsewidths due to the CPMG pulse train, the ${ }^{15} \mathrm{~N}$ (CPMG) pulse is calibrated using a ${ }^{15} \mathrm{~N}$ CPMG sequence where a gradient, an extra pulse to be calibrated, and a strong gradient are applied immediately after the CPMG pulse train. At this point in the sequence a density element 
proportional to either $N_{\mathrm{z}}, 2 N_{\mathrm{z}} H_{\mathrm{z}}$, or $N_{\mathrm{z}}\left(1-2 H_{\mathrm{z}}\right)$ is present depending of the type of CPMG experiment and the ${ }^{15} \mathrm{~N}$ pulsewidth can therefore be calibrated in the usual manner and this is performed for different $v_{C P M G}$ values. If the ${ }^{15} \mathrm{~N}$ pulsewidth depends on $v_{C P M G}$, CPMG pulse sequences that keep the load on the probe constant for various $v_{C P M G}$ values should be used in order to keep the pulsewidth constant.

4. Increasing the $T_{C P M G}$ delay allows for a greater number of exchange events to occur leading to a greater change in signal intensity as a function of $v_{C P M G}$ and also allowing for a larger range of $v_{C P M G}$ values as the lowest possible $v_{C P M G}$ is $1 / T_{C P M G}$ (or $2 / T_{C P M G}$ based on sequence). However large $T_{C P M G}$ delays can lead to a large reduction in the $\mathrm{S} / \mathrm{N}$ due to relaxation losses making it difficult to quantitate the change in intensities. As a compromise it is common to choose a $T_{C P M G}$ delay where the peak intensity has reduced by half compared with the reference spectrum that has no $T_{C P M G}$ delay.

5. As a large number of pulses are applied during the $T_{C P M G}$ delay, a long interscan delay (d1) has to be used to avoid damaging the probe. Typical d1 values lie between 2.1 and $2.5 \mathrm{~s}$.

\section{Acknowledgements}

DFH and PV are grateful to Prof. Lewis E Kay (University of Toronto), Dr. Ranjith Muhandiram, Dr. Algirdas Velyvis, Dr. Dimitri Korzhnev, Dr. Vitali Tugarinov, Dr. Philipp Neudecker, Dr. Patrik Lundstrom and other members of the Kay lab who exposed them to many of the methods presented here. DFH acknowledges the Biotechnology and Biological Sciences Research Council UK (BBSRC) for financial support. PV is supported by generous startup grant from TCIS/TIFH.

\section{References}

[1] M. Karplus, Aspects of protein reaction dynamics: Deviations from simple behavior, J Phys Chem B, 104 (2000) 11-27.

[2] R.H. Austin, K.W. Beeson, L. Eisenstein, H. Frauenfelder, I.C. Gunsalus, Dynamics of ligand binding to myoglobin, Biochemistry, 14 (1975) 5355-5373.

[3] H. Frauenfelder, S.G. Sligar, P.G. Wolynes, The energy landscapes and motions of proteins, Science, 254 (1991) 1598-1603.

[4] A.G. Palmer, NMR characterization of the dynamics of biomacromolecules, Chem Rev, 104 (2004) 3623-3640. 
[5] A. Sekhar, L.E. Kay, NMR paves the way for atomic level descriptions of sparsely populated, transiently formed biomolecular conformers, Proc Natl Acad Sci U S A, 110 (2013) 12867-12874.

[6] D. Chandler, Introduction to Modern Statistical Mechanics, Oxford University Press, Oxford, 1987.

[7] J. Cavanagh, W.J. Fairbrother, A.G. Palmer, M. Rance, N.J. Skelton, Protein NMR Spectroscopy, Principles and Practice, 2nd ed., Academic Press, 2006.

[8] F.A. Mulder, N.R. Skrynnikov, B. Hon, F.W. Dahlquist, L.E. Kay, Measurement of slow (micros-ms) time scale dynamics in protein side chains by (15)N relaxation dispersion NMR spectroscopy: application to Asn and Gln residues in a cavity mutant of T4 lysozyme, J Am Chem Soc, 123 (2001) 967-975.

[9] A.G. Palmer, 3rd, M.J. Grey, C. Wang, Solution NMR spin relaxation methods for characterizing chemical exchange in high-molecular-weight systems, Methods Enzymol, 394 (2005) 430-465.

[10] D.M. Korzhnev, L.E. Kay, Probing invisible, low-populated States of protein molecules by relaxation dispersion NMR spectroscopy: an application to protein folding, Acc Chem Res, 41 (2008) 442-451.

[11] D.F. Hansen, P. Vallurupalli, P. Lundstrom, P. Neudecker, L.E. Kay, Probing chemical shifts of invisible states of proteins with relaxation dispersion NMR spectroscopy: how well can we do?, J Am Chem Soc, 130 (2008) 2667-2675.

[12] P. Lundstrom, P. Vallurupalli, D.F. Hansen, L.E. Kay, Isotope labeling methods for studies of excited protein states by relaxation dispersion NMR spectroscopy, Nat Protoc, 4 (2009) 1641-1648.

[13] M.A. Mccoy, L. Mueller, Selective Shaped Pulse Decoupling in Nmr - Homonuclear [C13]Carbonyl Decoupling, J Am Chem Soc, 114 (1992) 2108-2112.

[14] H. Geen, R. Freeman, Band-Selective Radiofrequency Pulses, J Magn Reson, 93 (1991) 93-141.

[15] G.A. Morris, R. Freeman, Enhancement of Nuclear Magnetic-Resonance Signals by Polarization Transfer, J Am Chem Soc, 101 (1979) 760-762.

[16] L.E. Kay, P. Keifer, T. Saarinen, Pure Absorption Gradient Enhanced Heteronuclear Single Quantum Correlation Spectroscopy with Improved Sensitivity, J Am Chem Soc, 114 (1992) 10663-10665. 
[17] M. Ikura, L.E. Kay, M. Krinks, A. Bax, Triple-resonance multidimensional NMR study of calmodulin complexed with the binding domain of skeletal muscle myosin light-chain kinase: indication of a conformational change in the central helix, Biochemistry, 30 (1991) 5498-5504.

[18] M. Piotto, V. Saudek, V. Sklenar, Gradient-tailored excitation for single-quantum NMR spectroscopy of aqueous solutions, J Biomol NMR, 2 (1992) 661-665.

[19] P. Vallurupalli, D.F. Hansen, E. Stollar, E. Meirovitch, L.E. Kay, Measurement of bond vector orientations in invisible excited states of proteins, Proc Natl Acad Sci U S A, 104 (2007) 18473-18477.

[20] E. Kupce, R. Freeman, Optimized adiabatic pulses for wideband spin inversion, J Magn Reson A, 118 (1996) 299-303.

[21] D.F. Hansen, P. Vallurupalli, L.E. Kay, An improved $15 \mathrm{~N}$ relaxation dispersion experiment for the measurement of millisecond time-scale dynamics in proteins, $\mathrm{J}$ Phys Chem B, 112 (2008) 5898-5904.

[22] B. Jiang, B.H. Yu, X. Zhang, M.L. Liu, D.W. Yang, A N-15 CPMG relaxation dispersion experiment more resistant to resonance offset and pulse imperfection, $\mathbf{J}$ Magn Reson, 257 (2015) 1-7.

[23] A.J. Shaka, J. Keeler, T. Frenkiel, R. Freeman, An Improved Sequence for Broad-Band Decoupling - Waltz-16, J Magn Reson, 52 (1983) 335-338.

[24] R. Ishima, D.A. Torchia, Extending the range of amide proton relaxation dispersion experiments in proteins using a constant-time relaxation-compensated CPMG approach, $\mathbf{J}$ Biomol NMR, 25 (2003) 243-248.

[25] P. Vallurupalli, G. Bouvignies, L.E. Kay, Increasing the Exchange Time-Scale That Can Be Probed by CPMG Relaxation Dispersion NMR, J Phys Chem B, 115 (2011) 1489114900.

[26] P. Lundstrom, D.F. Hansen, L.E. Kay, Measurement of carbonyl chemical shifts of excited protein states by relaxation dispersion NMR spectroscopy: comparison between uniformly and selectively (13)C labeled samples, J Biomol NMR, 42 (2008) 35-47.

[27] P. Lundstrom, D.F. Hansen, P. Vallurupalli, L.E. Kay, Accurate measurement of alpha proton chemical shifts of excited protein states by relaxation dispersion NMR spectroscopy, $\mathrm{J}$ Am Chem Soc, 131 (2009) 1915-1926.

[28] E. Kupce, R. Freeman, Stretched adiabatic pulses for broadband spin inversion, J Magn Reson A, 117 (1995) 246-256. 
[29] E. Kupce, G. Wagner, Multisite band-selective decoupling in proteins, J Magn Reson B, 110 (1996) 309-312.

[30] E. Kupce, R. Freeman, Adiabatic Pulses for Wide-Band Inversion and Broad-Band Decoupling, J Magn Reson A, 115 (1995) 273-276.

[31] L.R. Brown, B.C. Sanctuary, Hetero-Tocsy Experiments with Waltz and Dipsi Mixing Sequences, J Magn Reson, 91 (1991) 413-421.

[32] V.Y. Orekhov, D.M. Korzhnev, L.E. Kay, Double- and zero-quantum NMR relaxation dispersion experiments sampling millisecond time scale dynamics in proteins, J Am Chem Soc, 126 (2004) 1886-1891.

[33] F. Delaglio, S. Grzesiek, G.W. Vuister, G. Zhu, J. Pfeifer, A. Bax, NMRPipe - a Multidimensional Spectral Processing System Based on Unix Pipes, J Biomol NMR, 6 (1995) 277-293.

[34] T.D. Goddard, D.G. Kneller, SPARKY 3 University of California, San Francisco.

[35] S. Kristensen, D. Hansen, Fuda: A function and data fitting and analysis package. , in, 2005.

[36] A. Ahlner, M. Carlsson, B.H. Jonsson, P. Lundstrom, PINT: a software for integration of peak volumes and extraction of relaxation rates, J Biomol NMR, 56 (2013) 191-202.

[37] D.M. Korzhneva, I.V. Ibraghimov, M. Billeter, V.Y. Orekhov, MUNIN: application of three-way decomposition to the analysis of heteronuclear NMR relaxation data, J Biomol NMR, 21 (2001) 263-268.

[38] A. Sekhar, P. Vallurupalli, L.E. Kay, Folding of the four-helix bundle FF domain from a compact on-pathway intermediate state is governed predominantly by water motion, Proc Natl Acad Sci U S A, 109 19268-19273.

[39] D.F. Hansen, CATIA (http://www.biochem.ucl.ac.uk/hansen/catia/).

[40] G. Bouvignies, Chemex (https://github.com/gbouvignies/chemex/releases), 2012.

[41] H.M. McConnell, Reaction Rates by Nuclear Magnetic Resonance, J Chem Phys, 28 (1958) 430-431 .

[42] F.A. Mulder, A. Mittermaier, B. Hon, F.W. Dahlquist, L.E. Kay, Studying excited states of proteins by NMR spectroscopy, Nat Struct Biol, 8 (2001) 932-935.

[43] D.M. Korzhnev, X. Salvatella, M. Vendruscolo, A.A. Di Nardo, A.R. Davidson, C.M. Dobson, L.E. Kay, Low-populated folding intermediates of Fyn SH3 characterized by relaxation dispersion NMR, Nature, 430 (2004) 586-590. 
[44] P. Vallurupalli, L.E. Kay, Complementarity of ensemble and single-molecule measures of protein motion: a relaxation dispersion NMR study of an enzyme complex, Proc Natl Acad Sci U S A, 103 (2006) 11910-11915.

[45] P. Vallurupalli, G. Bouvignies, L.E. Kay, Studying "invisible" excited protein States in slow exchange with a major state conformation, J Am Chem Soc, 134 (2012) 8148-8161.

[46] N.L. Fawzi, J. Ying, R. Ghirlando, D.A. Torchia, G.M. Clore, Atomic-resolution dynamics on the surface of amyloid-beta protofibrils probed by solution NMR, Nature, 480 (2011) 268-272.

[47] A.G. Palmer, F. Massi, Characterization of the dynamics of biomacromolecules using rotating-frame spin relaxation NMR spectroscopy, Chem Rev, 106 (2006) 1700-1719.

[48] N.R. Skrynnikov, F.W. Dahlquist, L.E. Kay, Reconstructing NMR spectra of "invisible" excited protein states using HSQC and HMQC experiments, J Am Chem Soc, 124 (2002) 12352-12360.

[49] R. Auer, D.F. Hansen, P. Neudecker, D.M. Korzhnev, D.R. Muhandiram, R. Konrat, L.E. Kay, Measurement of signs of chemical shift differences between ground and excited protein states: a comparison between $\mathrm{H}(\mathrm{S} / \mathrm{M}) \mathrm{QC}$ and R1rho methods, J Biomol NMR, 46 205-216.

[50] K. Tamiola, B. Acar, F.A. Mulder, Sequence-specific random coil chemical shifts of intrinsically disordered proteins, J Am Chem Soc, 132 (2010) 18000-18003.

[51] C. Sanchez-Medina, A. Sekhar, P. Vallurupalli, M. Cerminara, V. Munoz, L.E. Kay, Probing the free energy landscape of the fast-folding gpW protein by relaxation dispersion NMR, J Am Chem Soc, 136 (2014) 7444-7451.

[52] Y. Shen, F. Delaglio, G. Cornilescu, A. Bax, TALOS+: a hybrid method for predicting protein backbone torsion angles from NMR chemical shifts, J Biomol NMR, 44 (2009) 213223.

[53] Y. Shen, A. Bax, Protein structural information derived from NMR chemical shift with the neural network program TALOS-N, Methods Mol Biol, 1260 (2015) 17-32.

[54] P. Vallurupalli, D.F. Hansen, L.E. Kay, Structures of invisible, excited protein states by relaxation dispersion NMR spectroscopy, Proc Natl Acad Sci U S A, 105 (2008) 1176611771.

[55] G. Bouvignies, P. Vallurupalli, D.F. Hansen, B.E. Correia, O. Lange, A. Bah, R.M. Vernon, F.W. Dahlquist, D. Baker, L.E. Kay, Solution structure of a minor and transiently formed state of a T4 lysozyme mutant, Nature, 477 (2011) 111-114. 
[56] D.M. Korzhnev, T.L. Religa, W. Banachewicz, A.R. Fersht, L.E. Kay, A transient and low-populated protein-folding intermediate at atomic resolution, Science, 329 (2010) 13121316.

[57] P. Neudecker, P. Robustelli, A. Cavalli, P. Walsh, P. Lundstrom, A. Zarrine-Afsar, S. Sharpe, M. Vendruscolo, L.E. Kay, Structure of an intermediate state in protein folding and aggregation, Science, 336 (2012) 362-366.

[58] Y. Shen, O. Lange, F. Delaglio, P. Rossi, J.M. Aramini, G.H. Liu, A. Eletsky, Y.B. Wu, K.K. Singarapu, A. Lemak, A. Ignatchenko, C.H. Arrowsmith, T. Szyperski, G.T. Montelione, D. Baker, A. Bax, Consistent blind protein structure generation from NMR chemical shift data, Proc Natl Acad Sci U S A, 105 (2008) 4685-4690.

[59] A. Cavalli, X. Salvatella, C.M. Dobson, M. Vendruscolo, Protein structure determination from NMR chemical shifts, Proceedings of the National Academy of Sciences of the United States of America, 104 (2007) 9615-9620.

[60] J.H. Prestegard, H.M. al-Hashimi, J.R. Tolman, NMR structures of biomolecules using field oriented media and residual dipolar couplings, Q Rev Biophys, 33 (2000) 371-424.

[61] A. Bax, G. Kontaxis, N. Tjandra, Dipolar couplings in macromolecular structure determination, Methods Enzymol, 339 (2001) 127-174.

[62] D.F. Hansen, P. Vallurupalli, L.E. Kay, Quantifying two-bond 1HN-13CO and one-bond 1H(alpha)-13C(alpha) dipolar couplings of invisible protein states by spin-state selective relaxation dispersion NMR spectroscopy, J Am Chem Soc, 130 (2008) 8397-8405.

[63] P. Vallurupalli, D.F. Hansen, L.E. Kay, Probing structure in invisible protein states with anisotropic NMR chemical shifts, J Am Chem Soc, 130 (2008) 2734-2735.

[64] N.R. Skrynnikov, F.A. Mulder, B. Hon, F.W. Dahlquist, L.E. Kay, Probing slow time scale dynamics at methyl-containing side chains in proteins by relaxation dispersion NMR measurements: application to methionine residues in a cavity mutant of T4 lysozyme, J Am Chem Soc, 123 (2001) 4556-4566.

[65] P. Lundstrom, H. Lin, L.E. Kay, Measuring 13Cbeta chemical shifts of invisible excited states in proteins by relaxation dispersion NMR spectroscopy, J Biomol NMR, 44 (2009) 139-155.

[66] D.F. Hansen, P. Neudecker, P. Vallurupalli, F.A. Mulder, L.E. Kay, Determination of Leu side-chain conformations in excited protein states by NMR relaxation dispersion, J Am Chem Soc, 132 (2010) 42-43. 
[67] D.F. Hansen, L.E. Kay, Determining valine side-chain rotamer conformations in proteins from methyl 13C chemical shifts: application to the $360 \mathrm{kDa}$ half-proteasome, J Am Chem Soc, 133 (2011) 8272-8281.

[68] S. Hiller, H. Arthanari, G. Wagner, The T-lock: automated compensation of radiofrequency induced sample heating, J Biomol NMR, 44 (2009) 69-76.

[69] C. Ammann, P. Meier, A.E. Merbach, A Simple Multi-Nuclear Nmr Thermometer, J Magn Reson, 46 (1982) 319-321. 